\title{
Solving Bilevel Linear Multiobjective Programming Problems
}

\author{
Calice Olivier Pieume ${ }^{1}$, Patrice Marcotte ${ }^{2}$, Laure Pauline Fotso ${ }^{3}$, Patrick Siarry ${ }^{4}$ \\ ${ }^{1}$ Department of Mathematics, Faculty of Science, University of Yaounde I, Yaounde, Cameroon \\ ${ }^{2}$ Department of Computer Science and Operations Research, University of Montreal, Montreal, Canada \\ ${ }^{3}$ Department of Computer Sciences, Faculty of Science, University of Yaounde I, Yaounde, Cameroon \\ ${ }^{4}$ LISSI, Faculty of Sciences and Technology, University of Paris XII Val-de-Marne, Paris, France \\ E-mail: calice.oliver.pieume@umontreal.ca,marcotte@iro.umontreal.ca,lpfotso@ballstate.bsu.edu, \\ siarry@univ-paris12.fr \\ Received July 27, 2011; revised August 28, 2011; accepted September 10, 2011
}

\begin{abstract}
This study addresses bilevel linear multi-objective problem issues i.e. the special case of bilevel linear programming problems where each decision maker has several objective functions conflicting with each other. We introduce an artificial multi-objective linear programming problem of which resolution can permit to generate the whole feasible set of the upper level decisions. Based on this result and depending if the leader can evaluate or not his preferences for his different objective functions, two approaches for obtaining Paretooptimal solutions are presented.
\end{abstract}

Keywords: Multiobjective Programming, Bilevel Programming, Feasible Solution, Pareto-Optimal Solutions

\section{Introduction}

Bilevel programming problems occur in diverse applications, such as transportation, economics, ecology, engineering and others. They have been extensively studied in the literature [1-3]. However, when facing a realworld bilevel decision problem, the leader and the follower may have multiple conflict objectives that should be optimized simultaneously for achieving a solution [4]. There are only very few approaches in the literature dealing with bilevel multiobjective problems: less than a dozen of paper in the literature are related to this particular class of problems to our knowledge [5-8]. Three reasons at least can explain the fact that the issue has not yet received a broad attention in the literature: the difficulty of searching and defining optimal solutions; the lower level optimization problem has a number of tradeoff optimal solutions; and it is computationally more complex than the conventional Multi-Objective Programming Problem or a bilevel Programming Problem. Consequently, it is extremely desirable to develop a simple and practical technique that can permit to find efficient solutions for this class of bilevel programming problem.
This study addresses linear multi-objective problem issues. The optimistic formulation is considered. We introduced an artificial multi-objective linear programming problem of which the resolution can permit to generate the whole set of feasible points of the upper level decisions. Based on this result and depending if the leader can evaluate or not his preferences for his different objective functions, two approaches for obtaining Pareto-optimal solutions are presented.

The paper is organized as follows. In the next section, we recall some notions about the solving of multiobjective programming problems (BLMPP). In Section 3, the optimistic formulation of a bilevel linear multi-objective programming problem is presented. Section 4 presents a relation between the feasible set of the upper level decisions and the Pareto-optimal set of a particular multiobjective programming problem introduced. Section 5 presents two approaches for solving BLMPP, based on the new relation established. Finally, the paper is concluded in Section 6.

\section{Efficient Points in Multiobjective Programming}

A multi-objective programming problem is formulated in 
general as follows:

$$
\begin{aligned}
& \min _{x} h(x)= \\
s, t \quad & \left(h_{1}(x), h_{2}(x), \cdots, h_{Q}(x)\right) \\
& x \in U
\end{aligned}
$$

where $h: R^{n} \rightarrow R^{Q}$ is the objective function vector and $U \subseteq R^{n}$ the set of constraints.

Due to the fact that, for $\mathbf{Q} \geq \mathbf{2}$, there is no canonical (total) order in $\mathbf{R}^{\mathbf{Q}}$, as there is on $\mathbf{R}$, one has to define how objective function vector

$$
\left(h_{1}(x), h_{2}(x), \cdots, h_{Q}(x)\right)
$$

must be compared for different alternatives $x \in U$. Closed pointed convex cones are generally used for the derivation of partial orders in the decision space. Let $K$ be an arbitrary cone such that $K \subset \mathbf{R}^{\mathbf{Q}}$, then the binary relation with respect to the cone $K$ (denoted $\leq_{K}$ ) is defined by:

$$
a \leq_{K} b \text { if and only if } b-a \in K
$$

Due to the fact that it could not be possible to find a solution that optimizes simultaneously all the objective functions, a weaker concept, the concept of Non-dominated point is used.

\section{Definition 1.}

A point $y_{0} \in h(U)$ is a non-dominated point with respect to the cone $K$ if and only if there does not exist a point $y \in h(U), y \neq y_{0}$, such that $y \leq_{K} y_{0}$. If $y^{*}$ is a non-dominated point with respect to the cone $K$, then $x^{*} \in U$, such that $y^{*}=h\left(x^{*}\right)$ is called Pareto-optimal (or efficient) solution with respect to the cone $K$.

The following definition of efficient points is the most used in the literature [9-11].

\section{Definition 2.}

A feasible point $x^{*} \in U$ is called Pareto-optimal if there does not exist $x \in U$ such that

$$
\left(h_{1}(x), h_{2}(x), \cdots, h_{Q}(x)\right) \leq\left(h_{1}\left(x^{*}\right), h_{2}\left(x^{*}\right), \cdots, h_{Q}\left(x^{*}\right)\right)
$$

and

$$
\left(h_{1}(x), h_{2}(x), \cdots, h_{Q}(x)\right) \neq\left(h_{1}\left(x^{*}\right), h_{2}\left(x^{*}\right), \cdots, h_{Q}\left(x^{*}\right)\right)
$$

If $x^{*}$ is Pareto-optimal, then $h\left(x^{*}\right)$ is called nondominated point.

Let us remark that Definition 2 is a particular case of Definition 1, where the cone used is $R_{+}^{Q} \backslash\left\{0_{Q}\right\}$. Paretooptimal points are then solutions that cannot be improved in one objective function without deteriorating their performance in at least one of the other objective functions. Through the paper, the set of efficient points of a multiobjective optimization problem defined by a vector function value $h$ on a feasible set $U$, with respect to a cone $K$, will be denoted by $E\left(h, U, \leq_{K}\right)$ and the corre- sponding non-dominated set denoted by $N\left(h, U, \leq_{K}\right)$.

Unfortunately, for a majority of $M O P P$, it is not easy to obtain an exact description of the efficient set, that typically includes a very large or infinite number of points. Solving multiobjective programming problems consists in general to find a finite subset of the efficient set and present them for evaluation to the decision maker $(D M)$. A set $W$ is a good representation of the efficient set $E\left(h, U, \leq_{K}\right)$ if the following three conditions are fulfilled: $W$ is finite and contain a reasonable number of points; non-dominated points corresponding to $W$ do not miss a large portion of $N\left(h, U, \leq_{K}\right)$ (coverage criterion); and these points do not include points that are very close to each other (uniformity criterion).

The coverage error is mathematically defined by:

$$
\varepsilon=\max _{x \in E\left(h, U, \leq_{K}\right)} \min _{y \in W} d(h(x), h(y))
$$

where $d(. .$.$) is a given distance defined in the decision$ space. This measure can be seen as the error associated to the worst representation of an element of $E\left(h, U, \leq_{K}\right)$ in $W$. The uniformity of a representation is mathematically defined by:

$$
\mu=\min _{y, z \in W ; y \neq z} \mathrm{~d}(h(y), h(z))
$$

It measures the distance between a pair of closed elements of $W$. A smaller number of points, a lower coverage error and a more uniform level are desirable in order to have a good representation of the efficient set. Such subsets are called representative subsets of the efficient set. Approaches that could generate a representative subset of the efficient set when solving linear multicriteria optimization problems, can be found in [9-11].

\section{Optimistic Formulation of a BLMPP}

A standard Bilevel Programming Problem (BPP) can be modeled as follows:

$$
\begin{aligned}
& \min _{x \in X} F(x, y) \\
& \text { subject to }\left\{\begin{array}{l}
G(x) \leq 0 \\
y \text { solve }\left\{\begin{array}{l}
\min _{y \in Y} f(x, y) \\
s, t \\
g(x, y) \leq 0
\end{array}\right.
\end{array}\right.
\end{aligned}
$$

where $x \in X \subset R^{n_{1}} ; y \in Y \subset R^{n_{2}} ; F, f: X \times Y \rightarrow R$ are the outer (planner's or leader's) problem objective function and the inner (behavioral or follower's) problem objective function, respectively; $G, g: X \times Y \rightarrow R$ are inequality constraints. $x($ resp $y)$ are decision variables controlled by the leader (resp the follower).

If $F$ and $f$ are vector value functions 


$$
\left(F: R^{n_{1}} \times R^{n_{2}} \rightarrow R^{m_{1}} \text { and } f: R^{n_{1}} \times R^{n_{2}} \rightarrow R^{m_{2}}\right),
$$

then one speak about bilevel multiobjective programming problems (BMPP). The standard formulation of a (BMPP) can then be as follows (Equation (1)):

Our focus will be on the linear formulation of a BMPP, given as follows:

$$
\begin{aligned}
& \min _{x \in R_{+}^{n_{1}}} F(x, y)=\left(C_{1}(x, y), C_{2}(x, y), \cdots, C_{m_{1}}(x, y)\right) \\
& s, t\left\{\begin{array}{l}
A_{1} x \leq b_{1} \\
y \text { solves }\left\{\begin{array}{l}
\min _{y \in R_{+}^{n_{2}}} f(x, y)=\left(c_{1} y, c_{2} y, \cdots, c_{m_{2}} y\right) \\
s-t \\
A_{2} x+A_{3} y \leq b_{2}
\end{array}\right.
\end{array}\right.
\end{aligned}
$$

(BLMPP)

where $C_{i}, i=1, \cdots, m_{1}$ are $n_{1}+n_{2}$-dimensional constant row vectors; $c_{i}, i=1, \cdots, m_{2}$ are $n_{2}$-dimensional constant row vectors; $b_{1}$ is a p-dimensional constant column vector and $b_{2}$ is a q-dimensional constant column vector; $A_{1}$ is a $p \times n_{1}$ constant matrix; $A_{2}$ is a $q \times n_{1}$ constant matrix and $A_{3}$ a $q \times n_{2}$ constant matrix.

Let us denote by $R(x)$, the set of rational responses of the follower, for each decision $x$ of the leader. It is defined as the Pareto-optimal point of the following problem:

$$
\begin{aligned}
& \min _{y \in R_{+}^{n_{2}}} f(x, y)=\left(c_{1} y, c_{2} y, \cdots, c_{m_{2}} y\right) \\
& \text { s.t } \quad A_{3} y \leq b_{2}-A_{2} x
\end{aligned}
$$

with this notation, one has the following formulation of BLMPP:

$$
\begin{aligned}
& \min _{x \in X} F(x, y)=\left(C_{1}(x, y), C_{2}(x, y), \cdots, C_{m_{1}}(x, y)\right) \\
& \text { Subject to }\left\{\begin{array}{l}
A_{1} x \leq b_{1} \\
y \in R(x)
\end{array}\right.
\end{aligned}
$$

Using also the following representation for the feasible space of BLMPP:

$$
\Omega=\left\{(x, y) \in R_{+}^{n_{1}} \times R_{+}^{n_{2}} / A_{1} x \leq b_{1} \text { and } y \in R(x)\right\}
$$

One obtains then the following optimistic formulation of BLMPP:

$$
\begin{aligned}
& \min _{x, y} F(x, y)=\left(C_{1}(x, y), C_{2}(x, y), \cdots, C_{m_{1}}(x, y)\right) \\
& \text { s.t } \\
& (x, y) \in \Omega
\end{aligned}
$$

(BMPP’)

We present in the following section a theoretical result that will be used after to derive two algorithms for solving BLMPP'. Through all the rest of the paper, $Z$ represents the set defined as follows:

$$
Z=\left\{(x, y) \in R_{+}^{n_{1}} \times R_{+}^{n_{2}} / A_{1} x \leq b_{1} \text { and } A_{2} x+A_{3} y \leq b_{2}\right\}
$$

It is assumed that $Z$ is a non-empty and bounded set over the convex polyhedron. We call $S$ the solution set of the problem BLMPP'.

\section{A New Characterization of the Feasible Set of a BLMPP}

We introduce a multi-objective programming problem of which efficient set is exactly equivalent to the feasible set of BLMPP'. A similar result has already been developed in [5], but with a different multiobjective programming problem. The result of the author is as follows. Consider the following multi-objective programming problem:

$$
\begin{aligned}
& \min _{x, y} \tilde{f}(x, y)=\left(c_{1} y, c_{2} y, \cdots, c_{m_{2}} y, x\right) \\
& \text { s.t } \\
& A_{1} x \leq b_{1} \\
& A_{2} x+A_{3} y \leq b_{2} \\
& x \geq 0, y \geq 0
\end{aligned}
$$

Let $K_{1}=R_{+}^{m_{2}} \backslash\left\{0_{m_{2}}\right\} \times\left\{0_{n_{1}}\right\}$ and $\Omega$ be as defined above. The following result holds:

Lemma 1. $\Omega=E\left(\tilde{f}, Z, \leq_{K_{1}}\right)$

The inconvenient of this result is that it is not easily applicable. In fact, there does not exist approaches developed in the literature for finding efficient points with respect to the particular cone $K_{1}=R_{+}^{m_{2}} \backslash\left\{0_{m_{2}}\right\} \times\left\{0_{n_{1}}\right\}$. Methods are usually for cones that have the form $R_{+}^{n} \backslash\left\{0_{n}\right\}, n \in R$. It is the reason why in [5], the author approximates the efficient set of MPP2 by the weakly efficient set.

$$
\begin{aligned}
& \min _{x \in X} F(x, y)=\left(F_{1}(x, y), F_{2}(x, y), \cdots, F_{m_{1}}(x, y)\right) \\
& y \text { solves }\left\{\begin{array}{l}
G(x) \leq 0 \\
\min _{y \in Y} f(x, y)=\left(f_{1}(x, y), f_{2}(x, y), \cdots, f_{m_{2}}(x, y)\right) \\
g(x, y) \leq 0
\end{array}\right.
\end{aligned}
$$


We introduce now a new relation that could be applied directly.

Let us consider the following multi-objective linear programming problem:

$$
\begin{aligned}
& \min _{x, y} H(x, y)=\left(\begin{array}{rr}
0 & c \\
-I & 0 \\
e^{t} & 0
\end{array}\right)\left(\begin{array}{l}
x \\
y
\end{array}\right) \\
& \text { s.t } \\
& A_{1} x \leq b_{1} \\
& A_{2} x+A_{3} y \leq b_{2} \\
& x \geq 0, y \geq 0
\end{aligned}
$$

(LMPP1)

where $c$ is a $m_{2} \times n_{2}$ matrix with rows $c_{i}^{\prime} s ; e$ is a vector having each entry equal to 1 and $I$ is an $n_{1} \times n_{1}$ identity matrix. Recall that each $c_{i}$ represents the row vector that defined the ith-objective function of the follower. Let $K_{2}=R_{+}^{m_{2}+n_{1}+1} \backslash\left\{0_{m_{2}+n_{1}+1}\right\}$, then the following result holds.

Theorem 1. $\Omega=E\left(H, Z, \leq_{K_{2}}\right)$

\section{Proof:}

$\Leftarrow$ Let us show that $E\left(H, Z, \leq_{K_{2}}\right) \subset \Omega$

Let $z=\left(x_{0}, y_{0}\right) \in E\left(H, Z, \leq_{K_{2}}\right)$, from the definition of $E\left(H, Z, \leq_{K_{2}}\right)$, one has naturally $A_{2} x_{0}+A_{3} y_{0} \leq b_{2}$ and $A_{1} x_{0} \leq b_{1}$. So, in order to show that $z \in \Omega$, it suffices to show that $y_{0} \in R\left(x_{0}\right)$. Let us suppose the contrary. Then there exist $\bar{y}$ such that: 1) $A_{2} x_{0}+A_{3} \bar{y} \leq b_{2}$ and 2) $\bar{y}$ dominate $y_{0}$.

Relation 2) is equivalent to

$$
\left(c_{1} \bar{y}, c_{2} \bar{y}, \cdots, c_{m_{2}} \bar{y}\right) \leq\left(c_{1} y_{0}, c_{2} y_{0}, \cdots, c_{m_{2}} y_{0}\right)
$$

with at least one $k \in\left(1, \cdots, m_{2}\right)$ such that $c_{k} \bar{y} \leq c_{k} y_{0}$.

Let now consider the point $z^{*}=\left(x_{0}, \bar{y}\right)$, we have:

$$
\begin{aligned}
H z^{*}= & \left(\begin{array}{cc}
0 & c \\
-I & 0 \\
e^{t} & 0
\end{array}\right)\left(\begin{array}{l}
x_{0} \\
\bar{y}
\end{array}\right)=\left(\begin{array}{c}
c \bar{y} \\
-x_{0} \\
e^{t} x_{0}
\end{array}\right) \text { and } \\
H z & =\left(\begin{array}{cc}
0 & c \\
-I & 0 \\
e^{t} & 0
\end{array}\right)\left(\begin{array}{l}
x_{0} \\
y_{0}
\end{array}\right)=\left(\begin{array}{c}
c y_{0} \\
-x_{0} \\
e^{t} x_{0}
\end{array}\right)
\end{aligned}
$$

Due to relation 2), one has $c \bar{y} \leq c y_{0}$ and $c \bar{y} \neq c y_{0}$, this permit to deduct that:

$$
H\left(\begin{array}{c}
x_{0} \\
\bar{y}
\end{array}\right) \leq H\left(\begin{array}{c}
x_{0} \\
y_{0}
\end{array}\right) \text { and } H\left(\begin{array}{c}
x_{0} \\
\bar{y}
\end{array}\right) \neq H\left(\begin{array}{c}
x_{0} \\
y_{0}
\end{array}\right)
$$

So $\left(x_{0}, \bar{y}\right)$ dominate $\left(x_{0}, y_{0}\right)$ with respect to the cone $K_{2}=R_{+}^{m_{2}+n_{1}+1} \backslash\left\{0_{m_{2}+n_{1}+1}\right\}$, which contradict the fact $\left(x_{0}, y_{0}\right)$ is a Pareto-optimal point with respect to the cone $K_{2}=R_{+}^{m_{2}+n_{1}+1} \backslash\left\{0_{m_{2}+n_{1}+1}\right\}$.

$\Rightarrow$ Let us show that $\Omega \subset E\left(H, Z, \leq_{K_{2}}\right)$

Suppose that there is $z=\left(x_{0}, y_{0}\right) \in \Omega$ such that $z \notin E\left(H, Z, \leq_{K_{2}}\right)$. Then there most be a point

$z_{1}=\left(x_{1}, y_{1}\right)$ such that $H z_{1}$ dominates $H z$. This implies that $\mathrm{Hz}_{1} \leq \mathrm{Hz}$ and $\mathrm{Hz}_{1} \neq \mathrm{Hz}$. Using the structure of the matrix $H$, and the fact that $z=\left(x_{0}, y_{0}\right)$ and $z_{1}=\left(x_{1}, y_{1}\right)$, one obtains:

$$
\left(\begin{array}{rr}
0 & c \\
-I & 0 \\
e^{t} & 0
\end{array}\right)\left(\begin{array}{l}
x_{1} \\
y_{1}
\end{array}\right)=\left(\begin{array}{c}
c y_{1} \\
-x_{1} \\
e^{t} x_{1}
\end{array}\right) \leq\left(\begin{array}{cc}
0 & c \\
-I & 0 \\
e^{t} & 0
\end{array}\right)\left(\begin{array}{l}
x_{0} \\
y_{0}
\end{array}\right)=\left(\begin{array}{c}
c y_{0} \\
-x_{0} \\
e^{t} x_{0}
\end{array}\right)
$$

and then

$$
\left(\begin{array}{c}
c y_{1} \\
-x_{1} \\
e^{t} x_{1}
\end{array}\right) \neq\left(\begin{array}{c}
c y_{0} \\
-x_{0} \\
e^{t} x_{0}
\end{array}\right)
$$

This implies that: $x_{1}-x_{0} \geq 0$ and $e^{t}\left(x_{1}-x_{0}\right) \leq 0$, which means that $x_{1}=x_{0}$ and also $e^{t} x_{1}=e^{t} x_{0}$. It follows that $c y_{1} \leq c y_{0}$ and $c y_{1} \neq c y_{0}$. This implies that $y_{1}$ dominates $y_{0}$. Contradicting the fact that $y_{0} \in R\left(x_{0}\right)$.

From this theorem, one can deduce that solving our problem (BLMPP') is equivalent to solve:

$$
\begin{aligned}
& \min _{x, y} F(x, y)=\left(C_{1}(x, y), C_{2}(x, y), \cdots, C_{m_{1}}(x, y)\right) \\
& \text { s.t } \\
& (x, y) \in E\left(H, Z, \leq_{K_{2}}\right)
\end{aligned}
$$

(BLMPP”)

The theorem led to the following corollary.

Corollary 1. $S=E\left(F, E\left(H, Z, \leq_{K_{2}}\right), \leq_{K_{1}}\right)$ where $K_{1}=R_{+}^{m_{1}} \backslash\left\{0_{m_{1}}\right\}$ and $K_{2}=R_{+}^{m_{2}+n_{1}+1} \backslash\left\{0_{m_{1}+m_{2}+1}\right\}$.

We present now two approaches for solving BLMPP' based on this last result.

\section{Two Approaches for Solving a BLMPP}

\subsection{First Approach}

Suppose that the upper decision maker is fully knowledgeable about all his preferences. One could then aggregate the leader objective functions using the weights $\lambda_{1}, \lambda_{2}, \cdots, \lambda_{m_{1}}$ that measure his preference concerning different objective functions. Solving BLMPP' will be then equivalent to solve the following problem:

$$
\begin{aligned}
& \min _{x, y} \sum_{i=1}^{m_{1}} \lambda_{m_{i}} C_{i}(x, y) \\
& \text { s.t }(x, y) \in E\left(H, Z, \leq_{K_{2}}\right)
\end{aligned}
$$


The obtained problem consists in an optimization of a linear function over a Pareto-optimal set. They are many approaches, developed in the literature, that are devoted to the optimization of a linear function over an efficient set (see [12], or the survey presented by Y. Yamamoto in [13] or C.O. Pieume and al in [14]). Any of these approaches can then be applied.

\subsection{Second Approach}

The second approach could be to generate a representative subset of $E\left(H, Z, \leq_{K_{2}}\right)$ using well known scheme [9-11], as described in the first section. Then one computes the image of the obtained subset by the leader objective functions and selects elements that led to non-dominated points for the leader. The following algorithm seems to be natural.

Step 1: Construct the following multiobjective linear programming problem:

$$
\begin{aligned}
& \min _{x, y} H(x, y)=\left(\begin{array}{rr}
0 & c \\
-I & 0 \\
e^{t} & 0
\end{array}\right)\left(\begin{array}{l}
x \\
y
\end{array}\right) \\
& \max _{x_{1}, x_{2}}\left(x_{1}+2 x_{2}, 3 x_{1}+x_{2}\right) \\
& s, t\left\{\begin{array}{l}
x_{1}+x_{2} \leq 3 \\
x_{1}, x_{2} \geq 0 \\
\text { where } y \text { solves }\left\{\begin{array}{l}
\max \\
y_{1}, y_{2} \\
\text { subje }
\end{array}\right.
\end{array}\right.
\end{aligned}
$$

The solution obtained by the authors [15] is the unique point $\left(x_{1}, x_{2}, y_{1}, y_{2}\right)=(1.5,1.5,4.1154,3.3846)$ with values of leader objective functions and follower objective functions respectively equal to $F^{*}=(4.5,6)$ and $f^{*}=(14.2692,11.6154)$.

The following Table 1 presents non dominated points provided by the second approach:

Consequently, Pareto-optimal points obtained are the points presented in the Table 2.

Figure 1 illustrates non-dominated points provided by the last approach (red points).

\section{Conclusions}

We have established in this paper equivalence between the feasible set of a bilevel multiobjective linear programming and the set of efficient points of an artificial

$$
\begin{aligned}
& s, t \\
& A_{1} x \leq b_{1} \\
& A_{2} x+A_{3} y \leq b_{2} \\
& x \geq 0, y \geq 0
\end{aligned}
$$

Step 2: Compute a representative subset (called $S$ ) of the efficient set of $L M P P 1$.

- $\quad$ For instance, approaches developed in [9-11] can be used.

Step 3: Compute the image set of $S$ by $F(Y=F(S))$.

Step 4: Find non-dominated points of $Y$ (called $Y_{\text {eff }}$ ) with respect to $F$.

Step 5: Find the Pareto-optimal points set $X_{E}$ corresponding to $Y_{\text {eff }}$.

- The Pareto-filter approach presented in [10] can be used in Step 4 and Step 5.

Step 6: $X_{E}$ is a representative subset of the efficient set of BMLPP', STOP.

\subsection{Illustrative Example}

Let consider the following problem [15]. multiobjective linear programming problem introduced. Two approaches have then been proposed in order to

\begin{tabular}{|c|c|c|c|c|c|c|c|c|}
\hline 0.2 .5 & 1.75 & 0. & 0 . & 0.9 & 1.0130208 & 0 . & 0.75 & 1.875 \\
\hline 3. 0.5 & 1.25 & 3. & 3. & 2.1 & 1.9435764 & 3. & 2.25 & 1.125 \\
\hline 6. 3.5 & 4.25 & 1 & 2.625 & 4.225 & 1.9696181 & 3.5625 & 5.25 & 3 \\
\hline 0.5. & 3.5 & 5. & 3.375 & 2.675 & 5.0434028 & 2.4375 & 1.5 & 0 \\
\hline
\end{tabular}
generate efficient points. The first approach aggregates the leader objective function and suggests to use a technique of optimization of linear function over an efficient

Table 1. Non dominated points.

\begin{tabular}{lrrrrrrrrr}
\hline 6. & 3.5 & 4.25 & 6. & 6. & 5.1 & 4.9001736 & 6. & 5.25 & 4.125 \\
\hline 3. & 8. & 6.5 & 3. & 3. & 4.8 & 4.9826389 & 3. & 4.5 & 6.75 \\
\hline
\end{tabular}

Table 2. Pareto-optimal points. 


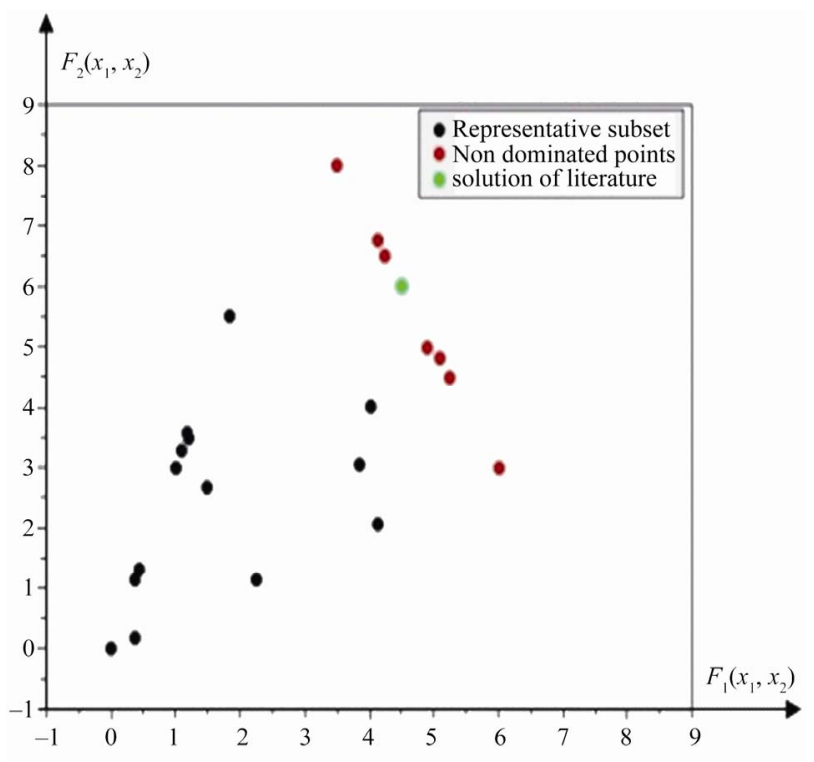

Figure 1. Representation of non dominated points.

set, in order to find an optimal solution. The second approach uses a Pareto-filter scheme to find an approximated discrete representation of the efficient set. The second approach has the advantage to keep the multicriteria concept of the upper DM, while the first one uses an aggregation process to eliminate the multi-criteria concept for the leader. We hope that this research can benefit the development of decision support systems for tackling bilevel multi-objective linear optimization problems in the real world.

\section{References}

[1] B. Colson, P. Marcotte and G. Savard, "An Overview of Bilevel Optimization,” Annals of Operational Research, Vol. 153, No. 1, 2007, pp. 235-256. doi:10.1007/s10479-007-0176-2

[2] J. Fulop, “On the Equivalence between a Linear Bilevel Programming Problem and Linear Optimization over the Efficient Set,” Technical Report WP93-1, Laboratory of Operations Research and Decision Systems, Computer and Automation Institute, Hungarian Academy of Sciences, 1993.

[3] C. O. Pieume, L. P. Fotso and P. Siarry, "A Method for Solving Bilevel Linear Programming Problem,” Journal of Information and Optimization Science, Vol. 29, No. 2,
2008, pp. 335-358.

[4] Y. Yin, "Multiobjective Bilevel Optimization for Transportation Planning and Management Problems," Journal of Advanced Transportation, Vol. 36, No. 1, 2000, pp. 93-105. doi:10.1002/atr.5670360106

[5] G. Eichfelder, "Multiobjective Bilevel Optimization," Mathematical Programming, Vol. 123, No. 2, 2008, pp. 419-449. doi:10.1007/s10107-008-0259-0

[6] D. Kalyanmoy and S. Ankur, "Solving Bilevel Multi-Objective Optimization Problems Using Evolutionary Algorithms,” KanGAL Report Number 2008005, 2008.

[7] I. Nishizaki and M. Sakawa, "Stackelberg Solutions to Multiobjective Two-Level Linear Programming Problems,” Journal of Optimization Theory and Applications, Vol. 103, No. 1, 1999, pp. 161-182. doi:10.1023/A:1021729618112

[8] X. Shi and H. Xia, "Interactive Bilevel Multi-Objective Decision Making,” Journal of the Operational Research Society, Vol. 48, No. 9, 1997, pp. 943-949.

[9] A. Messac and C. A. Mattson, "Generating Well Distributed Sets of Pareto Points for Engineering Using Physical Programming," Optimization and Engineering, Vol. 3, No. 4, 2002, pp. 431-450. doi:10.1023/A:1021179727569

[10] C. A. Mattson, A. A. Mullur and A. Messac, "Smart Pareto Filter: Obtaining a Minimal Representation of Multiobjective Design Space,” Engineering Optimization, Vol. 36, No. 6, 2004, pp. 721-740. doi:10.1080/0305215042000274942

[11] S. Sayin, "A Procedure to Find Discrete Representation of the Efficient Set with Specified Cover Errors," Operations Research, Vol. 51, No. 3, 2003, pp. 427-436. doi:10.1287/opre.51.3.427.14951

[12] H. P. Benson, "An All-Linear Programming Relaxation Algorithm for Optimizing over the Efficient Set,” Journal of Global Optimization, Vol. 1, No. 1, 1991, pp. 83-104. doi:10.1007/BF00120667

[13] Y. Yamamoto, "Optimization over the Efficient Set: Overview,” Journal of Global Optimization, Vol. 22, No. 1-4, 2002, pp. 285-317. doi:10.1023/A:1013875600711

[14] C. O. Pieume, L. P. Fotso and P. Siarry, "Finding Efficient Set in Multiobjective Linear Programming,” Journal of Information and Optimization Science, Vol. 29, No. 2, 2008, pp. 203-216.

[15] M. H. Farahi and E. Ansari, "A New Approach to Solve Multi-Objective Linear Bilevel Programming Problems,” Journal of Mathematics and Computer Science, Vol. 1, No. 4, 2010, pp. 313-320. 